\title{
A PSEUDO-EUCLIDEAN GEOMETRY
}

CURTIS M. FULTON

The axioms to be used in this paper are, with only two exceptions, the same as those for abstract Euclidean vector spaces. We denote the elements by small letters and call them points. The null element $\theta$, however, will not be considered a point. Capital letters will be used for scalars which are assumed to be real numbers. For addition and scalar multiplication we postulate the usual properties [1, pp. 3-4].

Our axioms for the "inner product," also a real number, are as follows:

$$
\begin{aligned}
(x+y, z) & =(x, z)+(y, z), \quad(A x, y)=A(x, y) ; \\
(x, y) & =-(y, x) ; \\
(x, y) & \neq 0 \quad \text { if } x, y \text { are linearly independent. }
\end{aligned}
$$

We agree to interpret $(x, y)$ as the directed distance between the points involved. If the distance between two distinct points vanishes we will speak of parallel points.

Let us first establish the somewhat surprising result that the "dimension number" is two. For this purpose we consider the equations

$$
\begin{aligned}
& (x, A x+B y+C z)=0, \\
& (y, A x+B y+C z)=0, \\
& (z, A x+B y+C z)=0
\end{aligned}
$$

for the unknowns $A, B, C$. Expanding these equations with the aid of (1) we see that their determinant is identically zero because of (2). Hence, there are solutions $A, B, C$ not all zero. Now, two cases arise. First, $A x+B y+C z \neq 0$. Clearly, $x, y$, and $z$, being dependent on this nonzero element on account of (3), are dependent. If they are points, these are all parallel. Second, the more important case, $A x+B y+C z$ $=\theta$. It is easily seen, that in this case $A: B: C=(y, z):(z, x):(x, y)$. Thus, we arrive at the fundamental identity for any three points that are not all parallel,

$$
(y, z) x+(z, x) y+(x, y) z=\theta .
$$

We now introduce a self-explanatory definition of straight line. Three points are defined to be collinear if and only if

Received by the editors August 25, 1958. 


$$
(y, z)+(z, x)+(x, y)=0
$$

and the distances involved are all different from zero. As a consequence of (4) and (5) we can represent a point $z$ on the line determined by $x$ and $y$ by

$$
z=R x+S y, \quad R+S=1 .
$$

A curious property of the line is obtained if we use a point $m$ at a distance $M$ from $x$ and $y$, that is $(m, x)=(m, y)=M \neq 0$. For a given $M$ we find the unique solution $m=M(x-y) /(x, y)$. From (6) we infer that $(m, z)=R(m, x)+S(m, y)=M$. In other words, all points of the line are on the circle of prescribed radius $M$. Conversely, if $(m, x)$ $=(m, y)=(m, z)=M$, we multiply (4) by $m$ and obtain (5).

Specializing by taking $M=1$ we find the point

$$
w=(x-y) /(x, y)
$$

which is characteristic for the line and will be called its L-point. It serves to write the equation of the line as $(w, x)=1$. Two lines whose equations are $(v, x)=1$ and $(w, x)=1$ have in general a point of intersection

$$
x=-(v-w) /(v, w) .
$$

If $(v, w)=0$ there is no common point and the lines are parallel. We also define $(v, w)$ as the directed angle between the lines.

At this point let $x, y, z$ be the vertices of a triangle (which term need not be clarified) and $u, v, w$ the $L$-points of the opposite sides. If we partition the triangle by means of a transversal it becomes obvious that the area may be defined as one half the oriented perimeter $(y, z)+(z, x)+(x, y)$. To illustrate further the nature of our geometry we develop a few trigonometric formulas. By the use of (7) we derive a formula for the area in terms of angle and two sides (which could be solved for the angle), namely

$$
(y, z)+(z, x)+(x, y)=(u, v)(y, z)(z, x) .
$$

From this we deduce immediately a formula similar to the ordinary law of sines,

$$
(u, v) /(x, y)=(v, w) /(y, z)=(w, u) /(z, x) .
$$

The two preceding relations or (8) yield

$$
(v, w)+(w, u)+(u, v)=(x, y)(v, w)(w, u) .
$$

The salient feature of our pseudo-Euclidean geometry is now apparent: unrestricted duality of point and line. It refers not only to 
incidence (and order) relations but also to the metric concepts of distance and angle. The theorems of Ceva and Menelaos which ordinarily are almost dual, are here exact duals if Ceva's theorem is stated in terms of angles.

It is convenient to introduce a coordinate system based on two fixed points $a, b$ such that $(a, b)=1$. The coordinates of a point $x$ are taken to be $X_{1}=(a, x), X_{2}=(b, x)$. Applying the identity (4) to $a, b, x$ we see that $x=-X_{2} a+X_{1} b$. For two points we are led to the distance formula

$$
(x, y)=X_{1} Y_{2}-X_{2} Y_{1} \text {. }
$$

Obviously the equation of a line will be linear. We omit the derivation of the equations for a change of coordinates. They are linear and homogeneous, the determinant being +1 . Hence the geometry could be described as the equiaffine plane without translations.

A model for our geometry is furnished by the Euclidean plane referred to Cartesian coordinates. The origin and all lines through it are not part of the model and correspond to "ideal" elements. Equation (9) plays the role of a nominal distance between two points.

Finally, we wish to thank the referee for a number of valuable suggestions.

\section{REFERENCE}

1. P. R. Halmos, Finite-dimensional vector spaces, 2d ed., D. Van Nostrand, 1958. University of California at Davis 